\title{
Towards a 3d Visualisation Framework for Construction Planning with Procession
}

\author{
Steve North \\ Virtual Reality Centre for the Built Environment \\ University College London WCIE 6BT UK \\ steve@stevenorth.com
}

Published as: North, S. (2000): ‘Towards a 3D Visualisation Framework for Construction Planning with Procession' in The Proceedings of IV2000 (IEEE International Conference on Information Visualization), E.Banissi, M.Bannatyne, C.Chen, F.Khosrowshahi, M. Sarfraz, A. Ursyn, Editors, USA: IEEE Computer Society, ISSN 1093-9547, ISBN 0-7695-0743-3, IEEE order no. PR00743, pp.577-582.

\begin{abstract}
The construction industry traditionally uses two dimensional visualisation techniques to analyse project progress. This usually takes the form of an 's-curve', so called because of the characteristic plot shape (anecdotally generated by resource application take-up in many projects). Analysing project progress in this manner provides a 'single view' guide to data trends. The building blocks of projects are 'tasks'. Deeper interpretation of a project might require studying individual s-curves for each task. Indicative patterns can go unrecognised in this mass of unfiltered visual data. 'Procession' is an information visualisation software tool. It has been developed to provide a more comprehensive representation of project progress, utilising a three-dimensional data surface. In addition to offering a 'single view' navigable data-surface, Procession also intelligently filters and emphasises tasks, according to the significance of their deviant performance parameters.
\end{abstract}

\section{Introduction}

The rationale for Procession's three-dimensional framework for construction planning is suggested by previous work on multi-dimensional morphological frameworks, in the field of systems engineering [1] [2]. Figure 1 shows the conceptual framework, with 'deviation parameters', 'deviation level' and 'tasks' as its three dimensions [3]. Deviation level represents units of time or money, which deviate from the flat terrain of the project baseline.

The three framework dimensions map to Procession's data surface, as follows:

- The $\mathrm{X}$ axis maps to the deviation parameters dimension

- The Y (height) axis maps to the deviation level dimension

- The $Z$ axis maps to the tasks dimension.

The values for $\mathrm{X}$ and $\mathrm{Z}$ define a two-dimensional plane, with $\mathrm{Y}$ providing the height scalar values.

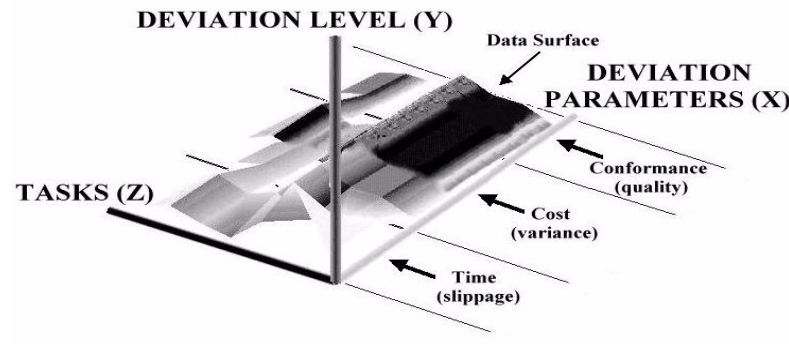

Figure 1. A 3d framework for construction planning, source: [3].

Therefore, a project with no deviations from the project baseline will produce a flat data surface. Procession's data surface is actually a 'carpet plot', generated by a scalar algorithm. The three dimensions are achieved from a twodimensional set of points, which are warped in the direction of the surface normal. The amount of warping is controlled by the scalar value. The point set is determined by the threedimensional framework's 'tasks' and 'deviation parameters' dimensions. The scalar (or height values) are provided by the framework's 'deviation level' dimension (see Figure 1). A fourth and non-spatial dimension is provided by colour mapping the data surface. This equates to the significance of the deviation level, as calculated by intelligent filtering and significance recognition.



Figure 2. An example of a navigable $3 d$ data surface, source: author. 


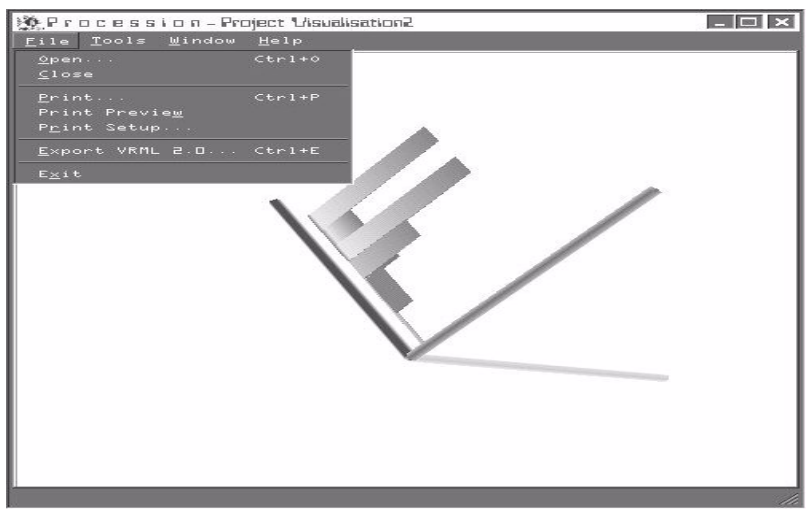

Figure 3. Procession's windows interface, source: author.

Procession is a stand-alone information visualisation [4] [5] application developed for the Microsoft Windows 95/98/2000/NT platform. It is capable of opening data files both from a local computer and from an Internet web server. Procession is an MDI (Multiple Document Interface) application (see Figure 3), which is to say that it can have several visualisation windows open simultaneously. Procession was developed in the $\mathrm{C}++$ language, utilising MFCs (Microsoft Foundation Classes).

Procession's three-dimensional graphics functionality is provided by VTK (the Visualization ToolKit), an 'open source' system providing a C++ class library, and a choice of Tcl/Tk, Java, and Python as interpreted languages [6].

\section{Methodological outline}

Current research is focusing on the evaluation of Procession. The main aim of this is to determine whether clients perceive Procession's progress reporting information to be more useful than currently utilised approaches. As a secondary aim, it is hoped that the evaluation will prove informative concerning the suitability of the conceptual three-dimensional framework. In order to evaluate Procession, comparative research will be conducted, making use of interview protocols and rapid prototyping. This evaluation is intended to determine a selected construction client's level of satisfaction with the quality and format of the project progress information provided.

In order that Procession's informational provision improves on the current model, a requirements capture stage is being undertaken. A planning data set has been obtained of a 'live' construction project. The researcher used this data as a skeleton to create simulated projects, with different sequences and outcomes. The purpose of this is to provide a testing ground for Procession, which must be unfamiliar to the construction client (there would be no motivation for information requests). The fictional project scenarios are based on a real nineteen-month project to refurbish flats and houses on an ex-local authority estate, in an inner-city area. The 'live' project objectives were: to provide new and clearly defined street patterns, to enlarge the individual gardens and to remove crime-ridden pedestrian routes. The total estate consists of 517 housing units (individual houses and flats). The 'live' project focuses on 252 of these units (123 houses and 129 flats).

Work on the houses will be conducted with most of the tenants in situ. A unique aspect of the project is the 'porch turnarounds' on the houses. This entails making the back of the house into a new entrance porch. The houses are given enlarged back gardens by gains made from old pedestrian ways. The new front gardens have parking spaces. Flats receive basic internal refurbishment. For example: kitchen fittings, vinyl kitchen flooring and decorations. Extensive landscaping is being conducted on the estate's communal areas. This includes turf laying, tree-planting, re-routing of gas mains and drains, new street patterns and improved estate lighting. Costs for the 'live' project were estimated from a construction industry standard pricing book [7]. The total budgeted cost before the project started was estimated as $£ 6,247,849$ (see Figure 4).

In order to generate project scenarios with a high level of realism, it was decided to identify real 'unacceptable' risks within the original budget and schedule. The two most serious risks were then used as a basis for 'problematic' issues, which were then fictionalised into the two scenarios. The identification of unacceptable risks was achieved using the risk analysis software tool Riskman Professional (see Section 3). When running through the research scenarios, construction clients taking the role of development workers will receive project progress reports at seven quarterly milestone intervals.

\begin{tabular}{|c|c|}
\hline myProject baseline budget at summary task level & \\
\hline SITE_SET_UP & \\
\hline SCAFFOLD_AND_TEMP_ROOFS_AND_REFURE_EXISTING_ROOFS_ON_SOME_HOUSES_AND_FLAT_ELOCKS & $\{20,907$ \\
\hline 'PORCH_TURNAROUNDS_ON__HOUSES_AND_INTERNAL_REFUREISHMENTS_ON_FLATS_GROUP1 & $\{2,585,299$ \\
\hline 'PORCH_TURNAROUNDS_ON_HOUSES_AND_INTERNAL_REFUREISHMENTS_ON_FLATS_GROUP2 & $\{412,588$ \\
\hline COMMISION_AND_CLEAR_AFTER_HOUSE_AND_FLAT_WORKS & $\{532,999$ \\
\hline LANDSCAPNN_TO_INDIVIDAL_HOUSE_AND_BLOCK_GARDENS & $\{2,091$ \\
\hline LANDSCAPNNG_ENTRE_SITE_AND_COMMUNAL_AREAS_AND_INTIAL_HORTICULTURE & $\{1,260,290$ \\
\hline CLEAN_RESIDENTIAL_AREAS_FOR_SECTIONAL_COMPLETION & $\{94,535$ \\
\hline SERVICES_TO_COMMON_AREAS & $£ 2,509$ \\
\hline SITE_HARD_LANDSCAPPNG_AND_INTIAL_WNORK_ON_ROADS_AND_PAVEMENT & $\{916,083$ \\
\hline MAIN_HORTICULTURAL_WORKS & $£ 133,190$ \\
\hline FINAL_MORKS_ON_ROADS_AND_PAVEMENTS & $\{98,316$ \\
\hline CLEAR_CLEAN_AND_FINAL_SITE_HAND-OVER & $£ 184,860$ \\
\hline & $£ 4,181$ \\
\hline & $\{6,247,849$ \\
\hline
\end{tabular}

\section{Figure 4. 'Live' project summary task budgets before commencement, source: author.}

Each of the simulated projects has had quarterly milestone files produced, to represent each of the progress reports throughout the project. These files will be stored on the researcher's laptop computer, in the CSV (Comma 
Separated Value) format. The milestone files compress a project of nineteen months into as many minutes. For this research, it has been decided that 'real time' simulation of a project is not practical. For example, a construction client's need for information concerning the progress of 'on-site' projects is likely to make up a vital, but proportionally small part of their workload. As the requirement for this information may happen at unpredictable times, it does not seem a sensible use of resources to attempt direct observations of the construction client's behaviour on a 'live' project. The prototypes will be evaluated by several different construction client volunteers.

The interview questions will consider the quality of information provided by the construction clients' current methods of progress reporting, allowing for later comparison with the prototypes. Construction clients will also be asked about their general familiarity with computers. After working with each prototype, construction clients will describe their perception of information quality. One protocol section will relate to functional aspects of the software prototype. Its purpose is to assess not the quality of the information that Procession presents, but the usability of the software. As such, reference will be made to standard guidelines for userinterface design.

In order to verify construction client's actual level of informational understanding, the data for each of the simulated projects will include one or more 'problematic issues' (i.e. an apparently trivial deviation factor that might result in catastrophic project failure). These issues will be specifically fictionalised to be more obviously detectable in Procession, than with traditional reporting methods. The protocol will contain questions relating to the construction clients' awareness of these hidden issues.

\section{Generating visualisation data from project scenarios}

In Section 2, the overall evaluation methodology was introduced. This section will now discuss in more detail the transformation of the original 'live' project data into a visualisation format suitable for Procession. Early on in the research sequence, a decision had been made to make Procession compatible with the market-leader project management package, Microsoft Project. Therefore, the 'live' project data was obtained in this format. The original project durations, dependencies and schedule were left unchanged.

In project management terminology, 'resources' are the materials and labour costs assigned to a specific task. Resources had not been assigned to the tasks in the original file and so assumptions were made about example resources that could be applied (i.e. the sub-tasks, materials and labour required to achieve the stated tasks). As the durations were pre-determined, resources based on hourly paid rates were not applicable. Instead, the labour and material costs were obtained from an industry standard pricing book [7] and calculated on a per house, block or site basis. In the initial version of the project budget, sums were applied as 'fixed cost' resource elements. Testing revealed that Earned Value (see Section 4) fields required for Procession seemed not to be functioning as expected when this approach was adopted (i.e. BCWS and BCWP values not present). Instead, a new baseline file was created with the 'cost' values cut and paste as 'fixed costs' directly attached to tasks (i.e. there were now no resources). Allocation of resources to tasks was done as units, rather than as a percentage. Number of available resource units was set to an arbitrary high figure $(10,000)$, to make sure that sufficient were available. Each 'fixed cost' was set to 'accrue at prorated'. 'Actual Costs' were calculated automatically by Microsoft Project. A 'Baseline' was saved. The baseline provides a record of all budget values before project commencement. These are then used for later analysis during the project lifecycle.

In order to generate possible scenarios from the data set, risk analysis (in the form of Monte-Carlo simulations) was applied to the Microsoft Project file. The Microsoft Project compatible software tool Riskman Professional (see www.riskdriver.com/riskmantool/) was used to identify potential project outcomes.

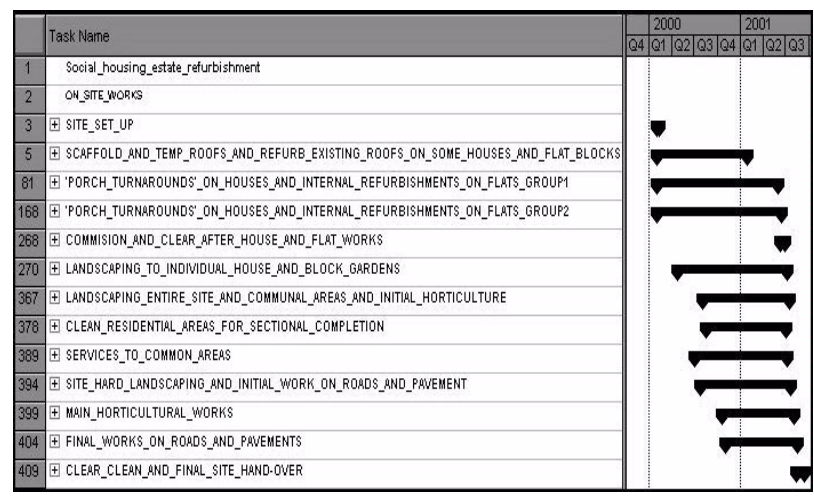

Figure 5. 'Live' project summary tasks and their scheduled durations before commencement, source: author.

Monte-Carlo simulations can be thought of as "statistical simulation methods, where statistical simulation is defined in quite general terms to be any method that utilizes sequences of random numbers to perform the simulation" [8]. First described during World War II's Manhattan Project, Monte-Carlo simulations are now widely used, both in hard science and for predicting games of chance [8]. Riskman uses Monte-Carlo simulations to analyse the likelihood and impact of specified risks. A project consists of tasks, related by precedence constraints (in project management terminology, a PERT network). In a simplified model, each task has a duration and a set of allocated resources. 
After completing a large number of simulation cycles, Riskman calculates each task's criticality to successful project outcome. Riskman outputs its results as Microsoft Excel charts and in its customised Microsoft Project 'Simulation View'. In the next section, this paper will go on to describe the results achieved when Riskman (and traditional project management visualisation techniques) were applied to the 'live' project data used for this research.

\section{Existing project visualisation methods}

The traditional method for reporting on project progress is the s-curve. This is generally utilised to analyse performance against budget in one of three ways. Most common of the three is 'earned value analysis' (see Figures 6 and 7). This provides the budgeted cost of completing the tasks actually finished at an observation point. This sum is the 'earned value'. Comparing this with the amount actually spent, is a good indicator of project performance. There is an acronym for each of the three curves plotted against each other for earned value analysis [9]:

- ACWP (Actual Cost of Work Performed. Actual cost of performing the completed tasks).

- BCWS (Budgeted Cost of Work Scheduled. The planned budget for the scheduled tasks).

- BCWP (Budgeted Cost of Work Performed. The planned budget for the tasks that were actually completedthe earned value).

In Completion Cost analysis (see Figures. 8 and 10), an s-curve plots three lines against each other to compare the original budgeted cost of the project and the revised cost, from the point of observation. There are three acronyms for these curves [9]:

- BAC (Budget At Completion. The planned budget).

- EAC (Estimate At Completion. The cost at completion revised as project progresses)

$=\mathrm{ACWP}+((\mathrm{BAC}-\mathrm{BCWP}) \times(\mathrm{ACWP} / \mathrm{BCWP}))$

- ETC (Estimate To Complete. The cost required to complete the project as at time ' $\mathrm{T}$ ')

$=(\mathrm{BAC}-\mathrm{BCWP}) \times(\mathrm{ACWP} / \mathrm{BCWP})$

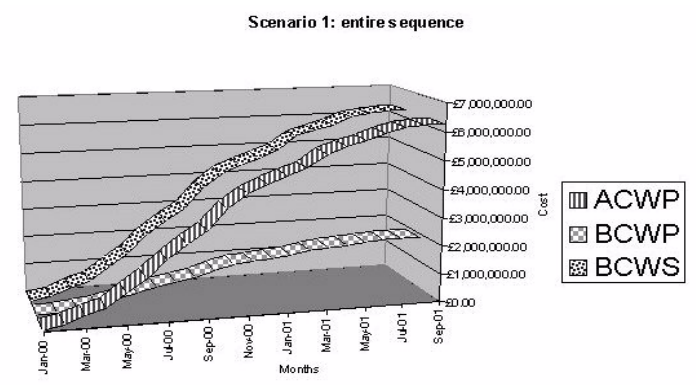

Figure 6. Scenario 1-entire project earned value s-curve, source: author.
Performance Indices provide a third approach to progress analysis. In this method, two curves are plotted (see Figures 9 and 11). One represents cost and the other schedule. For both plots, a flat horizontal line at $£ 1$ indicates that $£ 1$ expended is earning $£ 1$ of completed project. Values below $£ 1$, suggest that the project is either over-budget or behind schedule. In addition to providing 'to date' progress, the performance indices also indicate the underlying future trends in the project. The two curves represent:

- CPI (Cost Performance Index)

$=\mathrm{BCWP} / \mathrm{ACWP}$

- SPI (Schedule Performance Index)

$=\mathrm{BCWP} / \mathrm{BCWS}$

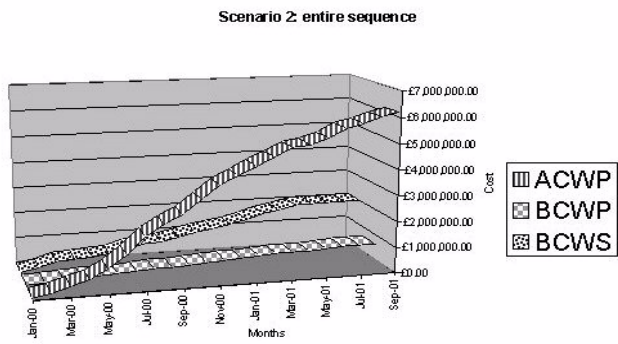

\section{Figure 7. Scenario 2-entire project earned value s-curve, source: author.}

\section{Conclusion}

The Monte-Carlo simulations revealed two summary tasks which were categorised as having attached risks of an 'unacceptable' level. The first of these was the summary task 'scaffolding and temporary roofs and refurbish existing roofs on some houses and flat blocks' (see Figures 4 and 5) which had the following unacceptable risks associated with it-

- Constraints on availability of external scaffolding components, impact the whole product.

- Requirements provided to scaffolding subcontractors are ambiguous.

- Sub-contracted scaffolding task disturbs project progress.

- Sub-contracted scaffolding task is badly managed and followed up.

- Workloads were under-estimated to gain contract.

- Required security level for the project is higher than usual because some tenants are remaining in occupation. Organisation of key access to occupied properties becomes expensive and time-consuming.

- Project is innovating in its field (porch turnarounds- see Section 2). 


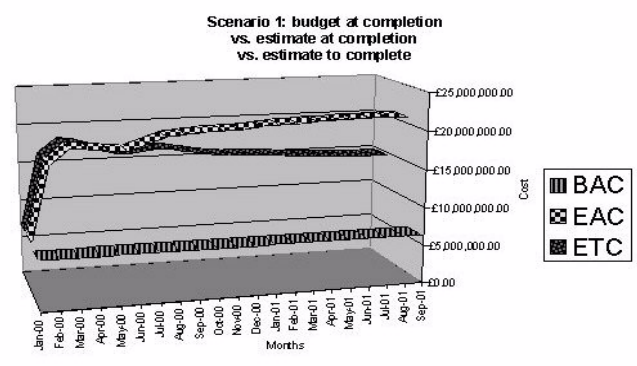

Figure 8. Scenario 1-entire project completion cost s-curve, source: author.

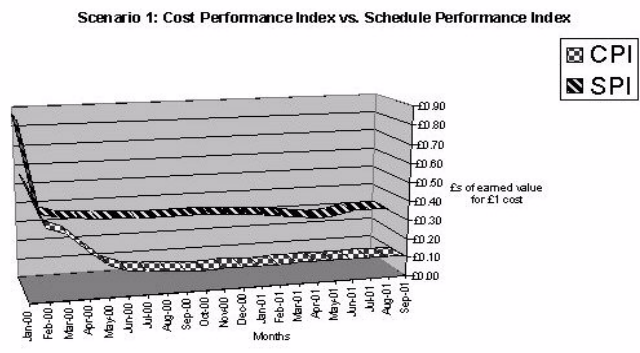

Figure 9. Scenario 1-entire project performance s-curve, source: author.

The second identified summary task was 'site hard landscaping and initial work on roads and pavements' (see Figures 4 and 5), which had the following unacceptable risks associated with it-

- Feasibility study on impact of road and service rerouting is missing.

- There is a low awareness of possible technical problems.

- When estimating costs, some activities are not quantifiable or have no reference in archived projects.

- Project risks were not assessed or are difficult to assess.

- Economical and political context has an influence on project and can be changed by unilateral decision at a high level. This particular project has to take particular regard of tenant opinion.

- Project environment is evolving.

- Technical difficulties are a real challenge.

- Detailed assessments of cost and time scale are not compatible with project budget and schedule. Planning does not take into account this fact and relies on false hypotheses.

The two unacceptable risks were fictionalised into scenarios (with embedded 'problematic' issues). In scenario 1 , the scaffolding sub-contractor failed to remove house scaffolding according to schedule, extending the duration of the roofing tasks and delaying the commencement of the porch turnaround work by almost three months. Scenario 2 sees the estate vehicle access restricted and most of the tenants remaining in residence. When the initial work on roads and pavements began in the third quarter, the situation became unmanageable. All works relating to the communal estate areas (roads, pavements, hard landscaping and horticulture) started to slip and their durations extended. As discussed in the last section, traditional visualisation techniques were used to analyse the entire sequence. A series of two-dimensional s-curves were produced. BAC (Budget At Completion), EAC (Estimate At Completion) and ETC (Estimate To Complete) were plotted against each other up to milestone 7 , as a cost/time s-curve. The expected total cost of the project (before it started) was $£ 6,247,849$. By the end of scenario 1's planned project time-scale, $£ 6,169,170.78$ had been spent and in order to complete (behind schedule), an additional $£ 14,533,790.31$ was required. The total project cost would then have spiralled to $£ 20,781,638.82$ (see Figure 8). In scenario $2,100 \%$ of the $£ 6,247,849$ predicted costs had been spent by the end of the planned project time-scale and in order to complete (behind schedule), an additional $£ 56,751,522.90$ was required. The total project cost would then have spiralled to $£ 62,920,693.68$ (see Figure 10).

CPI (Cost Performance Analysis) was plotted against SPI (Schedule Performance Analysis) as an s-curve to identify trends within the project progress. For scenario 1, the CPI and SPI showed that both the schedule and the budget trends were deteriorating rapidly before the first milestone. In the second quarter, both budget and schedule stabilised. By the third milestone, a slight improvement was seen in both curves, however they quickly dipped back and continued at approximately $£ 0.30$ for the rest of the schedule. At completion, the project was equally behind schedule and over budget (see Figure 9). Scenario 2 saw both the schedule and the budget plans deteriorating rapidly before the first milestone. In the second quarter, the budget stabilised, while the schedule continued to slip. By the third milestone, the schedule was stabilising and it

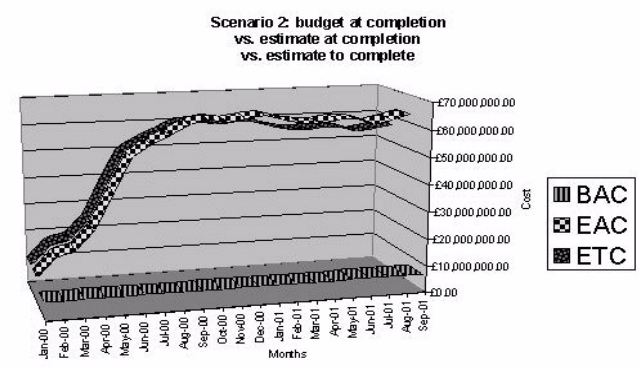

Figure 10. Scenario 2-entire project completion cost s-curve, source: author. 
continued to mirror the budget curve, with a differential of approximately $£ 0.20$. This continued until the scheduled completion date, with only a slight budget recovery in the last quarter. At completion, the project was still very behind schedule and over budget. The final CPI value reveals that $£ 0.10$ of value was being earned for every $£ 1$ spent (see Figure 11).

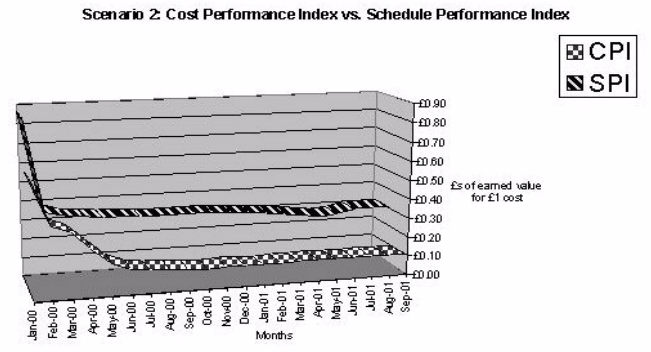

\section{Figure 11. Scenario 2-entire project performance s-curve, source: author.}

BCWS (Budgeted Cost of Work Scheduled) was plotted against BCWP (Budgeted Cost of Work Performed) and ACWP (Actual Cost of Work Performed) to produce an s-curve of Earned Value (see Section 4). For scenario 1, expenditure stayed almost as expected (ACWP and BCWS tracked each other throughout) but earned value (BCWP) slowly deteriorated throughout the sequence. Therefore, the work completed by the planned completion date had cost far more than budgeted and the schedule had proven to be over optimistic (see Figure 6). Throughout scenario 2, ACWP almost followed the budget in the baseline schedule, but increased in task durations. This resulted in the scheduled expenditure (BCWS) levelling out to match a longer time-scale. Despite slow improvement through the sequence, the earned value (BCWP) was so low compared with the ACWP, that the situation became irretrievable (see Figure 7).

The sequence outcome for scenario 1 was:

- Project had run out of time and budget to complete. No cost overruns had been incurred to date but more funds would be required to continue.

The sequence outcome for scenario 2 was:

- Project had run out of time and budget to complete. No cost overruns had been incurred to date but funds totalling many times the original budget would be required to continue.

Finally, two sets of seven milestone Microsoft Project files were generated, one for each scenario. To simulate progress at each milestone, changes were made to the tasks, in terms of their durations and percentages complete. For each scenario, it was necessary to work through the milestone files chronologically. This is because Microsoft Project always passes revised estimates onto the next milestone in the sequence. CSV data files for
Procession were exported from each of the Microsoft Project milestone files (i.e. 14 files total). In the next stage of the research, construction clients will apply the Procession initial prototype to the scenario milestone files.

\section{Acknowledgements}

This work was supported by a UK Office of Science \& Technology Foresight Challenge Award. The Virtual Reality Centre for the Built Environment is an interdisciplinary initiative between University College London, Imperial College and a consortium of UK construction industry partners.

\section{References}

[1] A.D. Hall, "Three-dimensional morphology of systems engineering", IEEE Transactions on System Science and Cybernetics, SSC-5(2), USA, 1969, pp.156-160.

[2] K. Pohl, "The Three Dimensions of Requirements Engineering: A Framework and Its Applications", Information Systems 19, 3, USA, 1994, pp.243-258.

[3] S. North, "Procession: using intelligent $3 \mathrm{~d}$ information visualisation to support client understanding during construction projects", Visual Data Exploration and Analysis VII, Robert F. Erbacher, Philip C. Chen, Jonathan C. Roberts, Craig M. Wittenbrink, (Editors), proceedings of SPIE (The International Society for Optical Engineering) Vol. $3690\left(24^{\text {th }}-26^{\text {th }}\right.$ January 2000, San Jose), ISSN 0277-786X, ISBN 0-8194-3578-3, USA, 2000, pp.356-364.

[4] S.K. Card, J.D. Macklinlay and B. Shneiderman, Readings in Information Visualization- using vision to think, Morgan Kaufmann, USA, 1999.

[5] C. Chen, Information Visualization and Virtual Environments, Springer-Verleg, USA, 1999.

[6] W. Shroeder, K. Martin, and B. Lorensen, The Visualization Toolkit, Prentice Hall, USA, 1998.

[7] Davis, Langdon and Everest, Spon's Architects' and Builders' Price Book, one hundred and twenty fifth edition, Davis Langdon \& Everest (Editors), E. \& F.N. Spon, UK, 2000.

[8] CSEP, "Introduction to monte-carlo methods", The Computational Science Education Project, http://csep1.phy.ornl.gov/CSEP/MC/MC.html, USA, 1995.

[9] K. Forsberg, H. Mooz and H. Cotterman, Visualizing Project Management, John Wiley \& Sons, USA, 1996. 